\title{
Management and outcomes of patients hospitalized with severe COVID-19 at a tertiary care center in midwestern United States
}

\author{
Sandeep Gupta ${ }^{1}$, Ashlesha Kaushik ${ }^{2}$, Jitendra Gupta ${ }^{1}$ \\ ${ }^{1}$ Division of Pulmonary, Critical Care and Sleep Medicine; ${ }^{2}$ Pediatric Infectious Diseases, Unity Point Health at St. Luke's \\ Regional Medical Center, Sioux City, IA, USA
}

\begin{abstract}
Knowledge of treatment regimens and outcomes for novel coronavirus disease 2019 (COVID-19) is evolving. Recent studies have reported mortality rates ranging from $39-50 \%$ among hospitalized patients with COVID-19. We report our experience of management and outcomes of hospitalized patients with COVID-19 at a large tertiary-care center in Midwestern United States. Of 658 patients presenting to our tertiary care center, 217 needed hospitalization, majority (77\%) of whom were severely sick requiring admission to the intensive care unit (ICU). All received corticosteroids, and 78\% of the patients received tocilizumab. More than two-thirds of the patients received anticoagulation and $80 \%$ of patients in the ICU had prone-positioning. The median duration of hospitalization was 12 days (interquartile range, 8 to16), median duration of intensive care unit stay was 7 days (interquartile range, 5 to 9) and requirement of mechanical ventilation was 6 days (interquartile range, 5 to 8 ) in our cohort. Of the 217 patients, 27 died (12\% mortality).
\end{abstract}

Correspondence: Sandeep Gupta, Division of Pulmonary, Critical Care and Sleep Medicine, Unity Point Health at St. Luke's Regional Medical Center, 2720 Stone Park Blvd, Sioux City, 51104 IA, USA.

Tel. +001.712.279-3500 - Fax: +001.712.255-4862.

E-mail: sandynoida@gmail.com

Key words: Outcomes; hospitalized; management; SARS-CoV-2; COVID-19.

Conflicts of interest: The authors have no conflicts of interest to disclose.

Contributions: Concept and design: All authors; Acquisition, analysis, or interpretation of data: SG, AK, JG; Drafting of the manuscript: SG, $\mathrm{AK}, \mathrm{JG}$; Critical revision of the manuscript for important intellectual content: SG, AK, JG; Statistical analysis: SG, AK; Administrative, technical, or material support: SG, AK, JG.

Received for publication: 31 August 2020.

Accepted for publication: 9 September 2020.

${ }^{\circ}$ Copyright: the Author(s), 2020

Licensee PAGEPress, Italy

Monaldi Archives for Chest Disease 2020; 90:1592

doi: 10.4081/monaldi.2020.1592

This article is distributed under the terms of the Creative Commons Attribution Noncommercial License (by-nc 4.0) which permits any noncommercial use, distribution, and reproduction in any medium, provided the original author(s) and source are credited.
The majority of our patients received corticosteroids, tocilizumab, anticoagulation and prone positioning. While higher mortality rates of $>30 \%$ have been reported in various studies among hospitalized patients with COVID-19, the majority of hospitalized patients in our cohort survived with a low mortality rate.

\section{Dear Editor,}

We are in the midst of an unprecedented COVID-19 global pandemic caused by severe acute respiratory syndrome coronavirus 2 (SARS-CoV-2) with increasing number of cases and mortality across the world with 24,854,140 confirmed cases and 838,924 deaths globally according to the World Health Organization (WHO) as of August 30, 2020 [1]. In the United States, there have been 5,934,824 cases and 182,149 deaths [2]. Given the unprecedented magnitude of impact of COVID-19 on public health, reporting of treatment regimens and clinical outcomes are needed to understand optimal therapeutic approaches for management of this novel viral disease.

In two recent studies from the United States, mortality rates ranging from $39 \%-50 \%$ in hospitalized patients with COVID-19 have been reported [3,4]. These studies have also reported a median duration of hospitalization of 17 days, ICU stay of 14 days and a median duration of mechanical ventilation ranging from 10-18 days $[3,4]$. In one of the studies with an overall mortality rate of $39 \%, 72 \%$ patients were reported to receive hydroxychloroquine, $9 \%$ received remdesivir, $26 \%$ patients received corticosteroids and $17 \%$ received IL-6 receptor antagonists [4].

We would like to report our experience of management and clinical outcomes of patients hospitalized with positive SARS COV-2 testing between April 14, 2020 to July 21, 2020 at a large tertiary-care center in Midwestern United States, serving the tristate area of Iowa, South Dakota and Nebraska, that has been a hotspot for COVID-19 due to meat-packaging industries in the vicinity.

Of the 658 patients presenting with COVID-19 to our institution, 217 patients were hospitalized, majority (73\%) of whom were non-Hispanic and $51 \%$ of the patients were female. The mean $( \pm \mathrm{SD})$ age of the patients was $54( \pm 12)$ years and the mean Charlson-Deyo risk score was $2.5( \pm 0.9)$.

All $(100 \%)$ of the hospitalized patients received corticosteroids (24\% patients received dexamethasone, $48 \%$ methylprednisolone, $28 \%$ received prednisolone, respectively). Among other specific therapeutic agents, tocilizumab was utilized in $170(78 \%)$ patients; 48 (24\%) patients received remdesivir, $82(38 \%)$ received convalescent plasma and $32(15 \%)$ patients received hydroxychloroquine. Famotidine and vitamin D were used in 43 (20\%) and $61(28 \%)$ patients respectively, and $165(76 \%)$ patients required anticoagulation with enoxaparin. Of the hospitalized 
patients, 77\% (167) needed intensive care, of whom 66\% (110) required mechanical ventilation ( MV); 30\% needed high-flow nasal-cannula and 7 patients received non-invasive ventilation; $80 \%$ (134) of ICU patients had prone-positioning, $40 \%$ (67) needed vasopressors and $83(49 \%)$ needed inhaled pulmonary vasodilators. The median duration of hospitalization was 12 days (interquartile range, 8 to 16). The median duration of ICU stay was 7 days (interquartile range, 5 to 9) and median duration of MV was 6 days (interquartile range, 5-8) days. Of the 217 patients, 27 $(12 \%)$ patients died. Of the 658 patients presenting to our center, $95.9 \%$ survived with a low mortality rate of $4.1 \%$.

In contrast to previous studies $[3,4]$, most of the hospitalized patients in our cohort received corticosteroids (100\%) and tocilizumab $(78 \%)$ and the majority survived ( $88 \%)$. Moreover, the median duration of hospitalization, ICU stay and mechanical ventilation was lower in our patients. Recently, tocilizumab has been shown to be beneficial in critically ill COVID-19 patients [5], possibly given the presence of cytokine release storm that accompanies severe COVID-19 and dexamethasone has also been shown to reduce mortality in hospitalized patients with COVID-19 as a part of RECOVERY trial results [6], similar to our experience.

The majority of our patients received corticosteroids, IL-6 inhibitor tocilizumab, anticoagulation and prone positioning and we witnessed a lower mortality rate than reported in recent studies among hospitalized patients. These therapeutic strategies may be considered as important components of treatment regimens for severe COVID-19 in hospitalized patients. Reporting of clinical and epidemiologic data is imperative to help understand effective management of this novel viral illness that has become a global health challenge, and therapies will likely evolve as more scientific evidence becomes available.

\section{References}

1. World Health Organization novel Coronavirus 2019. Available from: https://www.who.int/emergencies/diseases/novel-coronavirus-2019. Last accessed: August 27, 2020.

2. Centers For Disease Control and Prevention (CDC) Coronavirus disease 2019 (COVID-19): Situation summary. Available from: https://www.cdc.gov/coronavirus/2019-ncov/cases-updates/ cases-in-us.html. Last accessed: August 27, 2020.

3. Bhatraju PK, Ghassemieh BJ, Nichols M, et al. Covid-19 in Critically Ill Patients in the Seattle Region - Case Series. N Engl J Med 2020;382:2012-2022. doi:10.1056/NEJMoa2004500.

4. Cummings MJ, Baldwin MR, Abrams D, et al. Epidemiology, clinical course, and outcomes of critically ill adults with COVID19 in New York City: a prospective cohort study. Lancet 2020;395:1763-1770. doi:10.1016/S0140-6736(20)31189-2.

5. Price CC, Altice FL, Shyr Y, et al. Tocilizumab treatment for Cytokine Release Syndrome in hospitalized COVID-19 patients: survival and clinical outcomes. Chest 2020;S00123692(20)31670-6. doi:10.1016/j.chest.2020.06.006.

6. Ledford H. Coronavirus breakthrough: dexamethasone is first drug shown to save lives. Nature 2020;582:469. doi:10.1038/ d41586-020-01824-5. 